\title{
10 Second Heart Rate Variability
}

\author{
E Hodgart, PW Macfarlane \\ Cardiovascular and Medical Sciences, University of Glasgow, Scotland
}

\begin{abstract}
The aim of this study was to investigate the use of 10 second measures of heart rate variability (HRV) as an alternative to measures derived from a $24 \mathrm{hr}$ recording.

Conventional 10s 12-lead ECGs were obtained together with $24 \mathrm{hr}$ ECGs from 39 healthy volunteers and 41 patients who had recently suffered an MI. The Glasgow Program was used to derive SDNN and the average of the absolute difference between consecutive $R R$ intervals (AAD). $24 \mathrm{hr}$ ECG measures of HRV were derived from a commercially available system.

The normal ranges for 10s SDNN and 10s AAD were similar but there were significant differences between healthy volunteers and MI patients (e.g. $46.65 \mathrm{~ms} v 18.74$ ms for mean $S D N N-p<0.001)$.

Furthermore, the 10s measures showed a high sensitivity for detecting abnormally low HRV in MI patients leading to the conclusion that there is potential clinical value in utilising short term measure of $H R V$.
\end{abstract}

\section{Introduction}

Conventionally, heart rate variability is assessed from a 24-hour (Holter) ECG recording. This may be essentially as a spin off when this type of ECG is recorded for other reasons such as detection of a paroxysmal arrhythmia. It is probably fair to say that very few cardiologists specifically ask for an assessment of heart rate variability as part of a cardiological assessment. Indeed, unless there is an arrhythmic problem, Holter electrocardiography is not routinely part of a cardiological assessment in many health care systems. However, low heart rate variability has been shown to have prognostic value in patients with a recent myocardial infarction [1] and in other groups of patients also [2,3]. Indeed, using a 2 minute rhythm strip, Dekker et al [4] showed that low heart rate variability could predict the risk of coronary heart disease and mortality from several causes. Thus, if a measure of heart rate variability from a 10 second ECG could be shown to be of clinical value, it could be obtained routinely along with a standard 12 lead ECG recording. For this reason, a small study was carried out to investigate 10 -second heart rate variability in healthy individuals in comparison to patients with a recent myocardial infarction.

\section{Methods}

Conventional 10-second 12 lead electrocardiograms were obtained from a group of apparently healthy volunteers recruited mainly from staff and medical students in Glasgow Royal Infirmary and from patients who had recently suffered a myocardial infarction. A Burdick Eclipse 850i electrocardiograph was used and ECGs were transferred to a Siemens/Draeger Megacare ECG Management System for storage and further processing.

The sinus initiated RR intervals were measured by the Glasgow Program [5] and subsequently used for the calculation of SDNN as well as the average of the absolute differences between consecutive pairs of sinus initiated RR intervals (AAD)[6]. With this approach, premature atrial contractions were not included in the calculation of these measures of heart rate variability.

In addition, a 24-hour ECG recording was obtained using a two channel Spacelabs Burdick 90208 analogue recorder. The recordings were replayed and processed on a Medilog Excel 2 system (Oxford Instruments, Old Woking, UK). Tapes with insufficient hours of monitoring ( $<18$ hours), excess artefact, poor quality recording or technical failure were excluded from the analysis. The tapes were edited by experienced physiological measurement technicians and 24-hour estimates of SDNN were obtained from the turnkey system.

The RR intervals from the 24-hour analyser were also transferred to a PC for further processing and calculation of the heart rate variability fraction [7] which provides an estimate of heart rate variability in the form of a percentage. For example, the normal limits of HRVF are from $35 \%$ to $71 \%$ [7].

The patients with myocardial infarction were followed up one month after hospital discharge at the cardiac rehabilitation clinic. In terms of an endpoint for this short study, which was undertaken as part of a medical undergraduate research project, death or coronary artery bypass grafting within one month of the recording were taken. 
Statistical analysis was undertaken using the Mann Whitney Test while Spearman's rank correlation was used to assess the strength of association between 10second and 24 hour measures of heart rate variability. Regression analysis was used to predict 24-hour measures from 10 second parameters. Survival analysis at one month follow up was performed using a Kaplan-Meier plot and a log rank test was then used to compare the survival times in the two groups being studied, one with high and one with low heart rate variability.

\section{Results}

All patients were consented according to the ethical standards of the local Health Board and of those initially consented, a number were excluded because of new onset atrial fibrillation, Holter problems etc. Ultimately, there were 39 healthy volunteers and 41 patients recruited. The median age for the volunteers was 22 years and for the patients it was 71 years.

The normal ranges for 10-second SDNN and 10second AAD were 14-187 ms and 14-152 ms respectively. There was a significant difference between the mean SDNN for volunteers, viz $46.65 \mathrm{~ms}$ and that for MI patients, viz $18.74 \mathrm{~ms}(\mathrm{p}<0.001)$. Similarly, there were significant differences for the values of AAD.

By using the 6th percentile of the normal range of $\mathrm{AAD}$ as the cut off value, i.e. by selecting a specificity of $94 \%$, the sensitivity of AAD as test of low HRV in the post MI patients was $49 \%$.

Table 1 shows the correlation between the heart rate variability fraction and 3 measures of heart rate variability, namely 10 second AAD, 10 second SDNN and the 24-hour SDNN. Clearly the best correlation is with the 24 hour estimates but nevertheless there was a significant correlation with the 10 -second measures. An attempt was made to predict 24-hour measures from the 10-second ECG. The 80 subjects were split into a training set $(n=60)$ and a test set $(n=20)$. On the basis of the training set, a regression equation was derived to link the 10-second and 24-hour estimates of SDNN. (Fig 1) This equation was then applied to the 20 subjects in the test set to obtain their predicted 24-hour SDNN which was then compared with the measured 24-hour SDNN. A simple paired $t$ test showed that there was no significant difference between observed and predicted values but on the other hand the $95 \%$ limits of agreement between observed and predicted were so wide as to make this approach of no value.
Table 1. Correlations between the various measures of heart rate variability.

\begin{tabular}{|c|c|c|c|}
\hline & $\begin{array}{ll}10 \mathrm{~s} & \mathrm{AAD} \\
(\mathrm{ms}) & \end{array}$ & $\begin{array}{l}\text { 10s SDNN } \\
(\mathrm{ms})\end{array}$ & $\begin{array}{l}\text { 24hr SDNN } \\
(\mathrm{ms})\end{array}$ \\
\hline $\begin{array}{l}\text { 10s SDNN } \\
(\mathrm{ms})\end{array}$ & $\begin{array}{l}0.954 \\
<0.001\end{array}$ & & \\
\hline $\begin{array}{l}\text { 24hr SDNN } \\
(\mathrm{ms})\end{array}$ & $\begin{array}{l}0.470 \\
<0.001\end{array}$ & $\begin{array}{l}0.469 \\
<0.001\end{array}$ & \\
\hline $\operatorname{HRVF}(\%)$ & $\begin{array}{l}0.384 \\
<0.001\end{array}$ & $\begin{array}{l}0.386 \\
<0.001\end{array}$ & $\begin{array}{l}0.844 \\
<0.001\end{array}$ \\
\hline
\end{tabular}

With respect to survival analysis, the Kaplan Meier curves (Fig 2) were plotted using the cut off determined from the lower limits of 96 percentile normal range of HRV. Given the short time interval for follow up, it was no surprise to find that there was no significant difference at one month between those with normal and those with low heart rate variability.

\section{Discussion and conclusions}

Part of the rationale for looking at short term heart rate variability was that, in the West of Scotland Coronary Prevention Study [8] the 10-second measures did have univariate predictive value with respect to the endpoint of fatal or non fatal myocardial infarction in a group of over 5,000 men who participated in this primary prevention study. Furthermore, in the Rotterdam Study of the Elderly [9] there was also evidence to suggest that the 10 second SDNN had some predictive value.

The present study shows that indeed the short term assessment of HRV can be used to show that patients with cardiovascular problems do indeed have a lower heart rate variability than normal individuals. However, it is a limitation of the study that the normal group used for development of normal ranges was much younger than the patient group. Younger individuals tend to have more extreme heart rate variability, i.e. they have extremely high values due to "juvenile" sinus arrhythmia but nevertheless there was still a wide spectrum of ages in the normal group. The use of the technique of 96 percentile ranges also is another valuable approach in constructing normal ranges in that it is not biased by a high mean value for HRV. The lower limit of normal still remains the second percentile of the range of values irrespective of the mean value. 


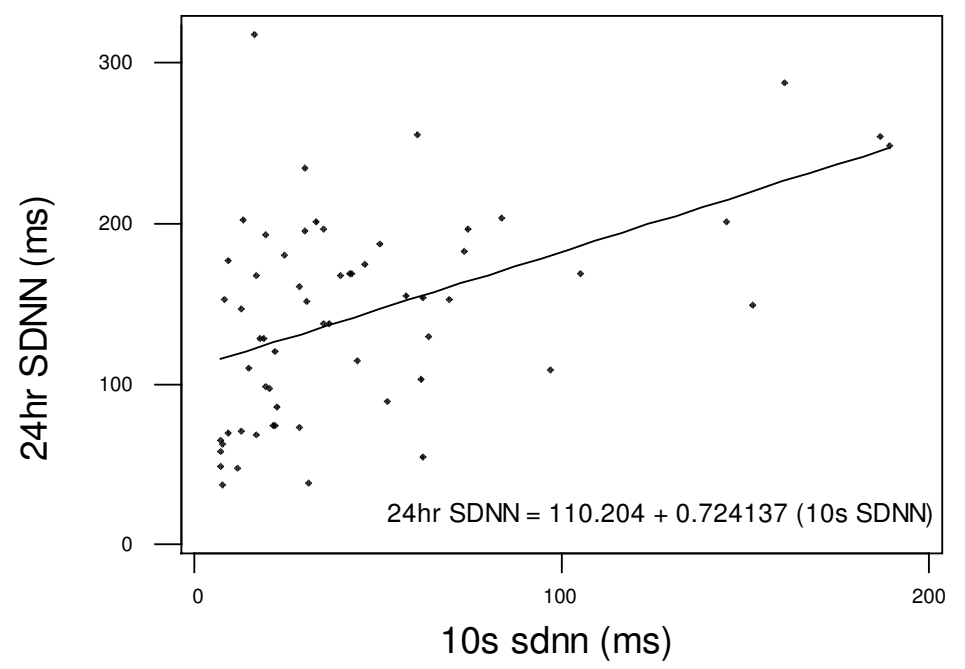

Fig. 1. Correlation 10s SDNN v. 24 hr SDNN.

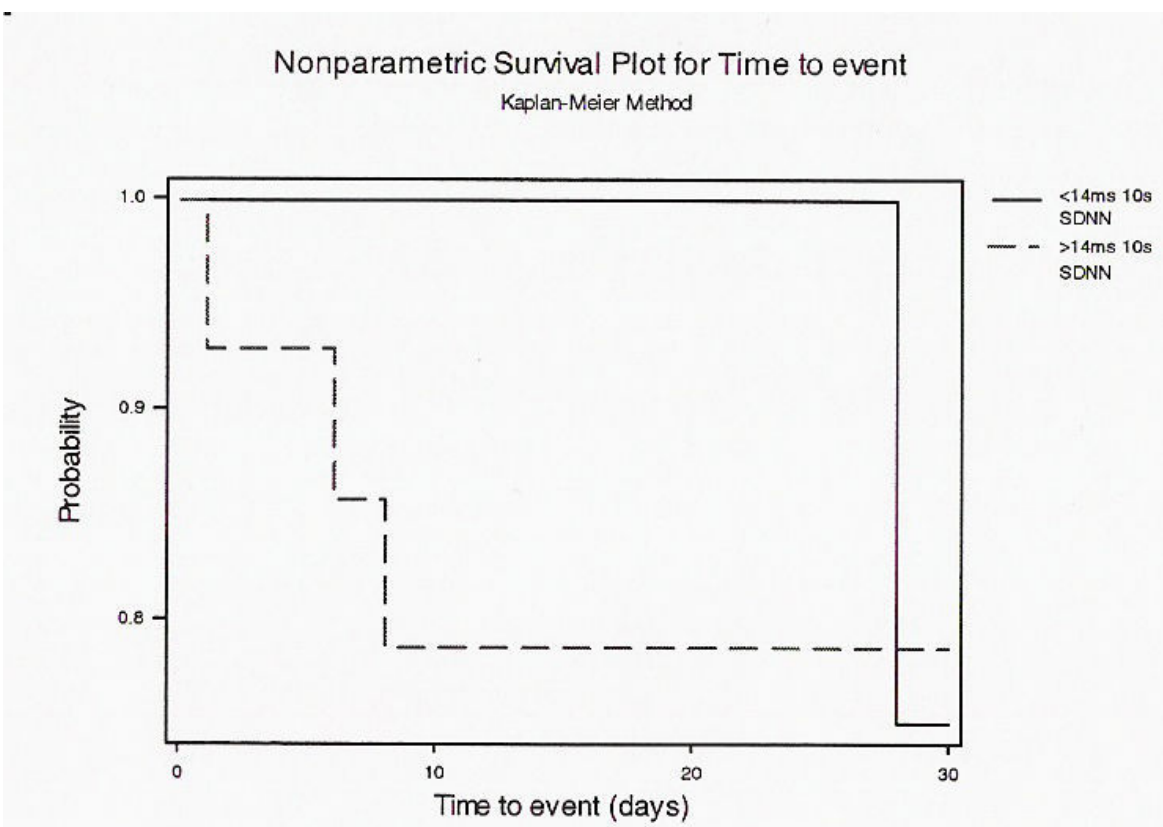

Fig. 2. 10s SDNN v. outcome at 30 days. 
Clearly, the use of the 10 second estimate would be of extreme benefit in assessing HRV as opposed to a need to record a 24-hour ECG. Undoubtedly, normal ranges for measures derived from different time periods are totally different but that is irrelevant if the appropriate range is applied. It does lead to questions of repeatability of indices and this has already been looked at elsewhere by the Glasgow Group [10].

In conclusion, this small study suggests there could be merit in first of all assessing 10-second measures of HRV in a larger healthy population and secondly applying results to patients with myocardial infarction who could be followed for a longer period of time.

\section{References}

[1] Kleiger RE, Miller JP, Bigger JT Jr, Moss AJ. Decreased heart rate variability and its association with increased mortality after acute myocardial infarction. Am J Cardiol 1987;59:256-262.

[2] Tsuji H, Larson MG, Venditti FJ et al. Impact of Reduced Heart Rate Variability on Risk for Cardiac Events. The Framingham Heart Study. Circulation 1996;94:2850-2855.

[3] Whitsel EA, Raghunathan TE, Pearce RM et al. RRinterval variation, the QT interval index and risk of primary cardiac arrest among patients without clinically recognised heart disease. Eur Heart J 2001;22:165.

[4] Dekker JM, Crow RS, Folsom AR et al. Low Heart Rate Variability in a 2-minute Rhythm Strip Predicts Risk of Coronary Heart Disease and Mortality from Several Causes. Circulation 2000;102:1239.

[5] Macfarlane PW, Devine B, Latif S, McLaughlin S, Shoat DB, Watts MP. Methodology of ECG interpretation in the Glasgow program. Meth Inform Med 1990;29:354-61.
[6] Julu POO, Hondo RG. Effects of atropine on autonomic indices based on electrocardiographic R-R intervals in healthy volunteers. $\mathrm{J}$ Neurol, Neurosurgery and Psychiatry 1992;55:31-35.

[7] Sosnowski M, Macfarlane PW, Cyz Z et al. Age adjustment of HRV measures and its prognostic value for risk assessment in patients late after myocardial infarction. Int J Cardiol 2002;86:249-58.

[8] Shepherd J, Cobbe SM, Ford I et al. Prevention of Coronary Heart Disease with Pravastatin in men with hypercholesterolemia. N Engl J Med 1995;333:13017.

[9] De Bruyne MC, Kors JA, Hoes AW et al. Both Decreased and Increased Heart Rate Variability on the Standard 10-second Electrocardiogram Predict Cardiac Mortality in the Elderly. The Rotterdam Study. Am J Cardiol 1999;150(12):1282-1288.

[10] Murray PG, Hamilton RM, Macfarlane PW. Reproducibility of a non invasive real time measure of cardiac parasympathetic actuity. Pysiol Meas 2001;22:661-672.

Address for correspondence

Professor P.W. Macfarlane

University Section of Cardiology and Exercise Medicine, Queen Elizabeth Building, Glasgow Royal Infirmary,

10 Alexandra Parade,

Glasgow G31 2ER

peter.w.macfarlane@clinmed.gla.ac.uk 